\title{
A Hybrid Fake Banknote Detection Model Using OCR, Face Recognition and Hough Features
}

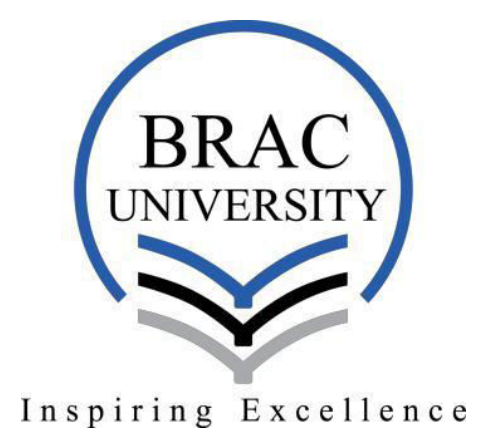

Bachelor of Computer Science and Engineering

Under the Supervision of

Dr. Jia Uddin

By

Adiba Zarin (13221030)

Ummay Tasnim (13321056)

Israt Jahan (13201018)

School of Engineering and Computer Science

BRAC University, Dhaka, Bangladesh

Submitted on: 13th August, 2018 


\section{Declaration}

We hereby declare that this thesis is based on results obtained from our own work. Materials of work found by other researcher are mentioned by reference. This thesis, neither in whole nor in part, has been previously submitted to any other University or Institute for the award of any degree or diploma.

Signature of Supervisor

Dr. Jia Uddin
Signature of the Authors

Adiba Zarin(13221030)

Ummay Tasnim(13321056)

Israt Jahan (13201018) 


\section{Acknowledgement}

First and foremost, we would like to thank Almighty Allah for enabling us to initiate the research, to put our best efforts and successfully conclude it.

Secondly, we submit our heartiest gratitude to our respected Supervisor Dr. Jia Uddin for his contribution, guidance and support in conducting the research and preparation of the report. Every last involvement of his, starting from instilling in us the deadliest of fears to the kindest words of inspiration has permitted us to effectively complete the paper. We are truly grateful to him.

We revere the patronage and moral support extended with love, by our parents as well as our friends. They helped us with their direct or indirect suggestions which aided in achieving our goal. We would also like to acknowledge the assistance we received from numerous resources over the Internet especially from fellow researchers' work.

Last but not the least; we thank BRAC University for providing us the opportunity of conducting this research and for giving us the chance to complete our Bachelor degree. 


\section{Table of Contents}

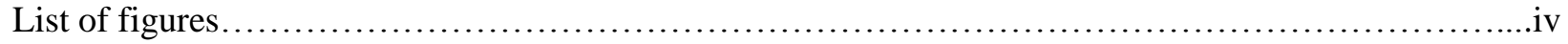

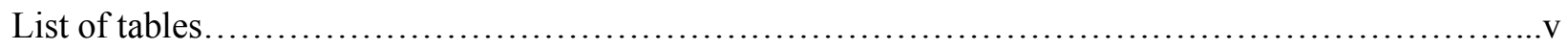

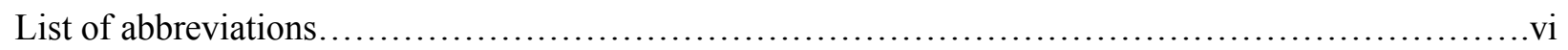

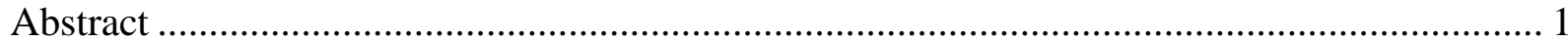

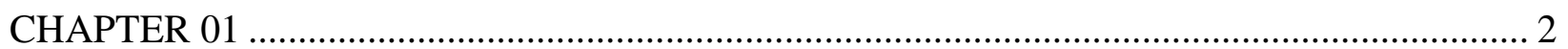

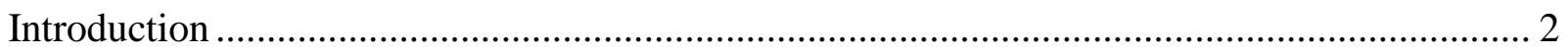

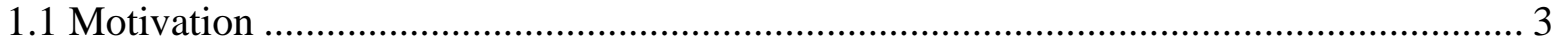

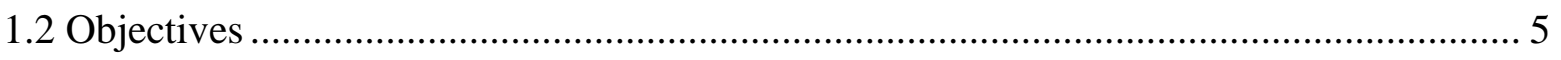

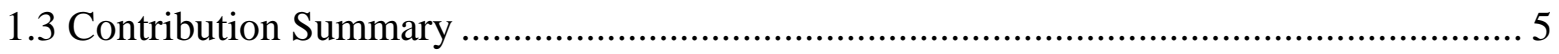

1.4 Thesis Outline

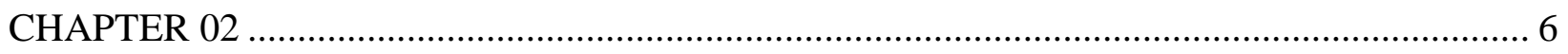

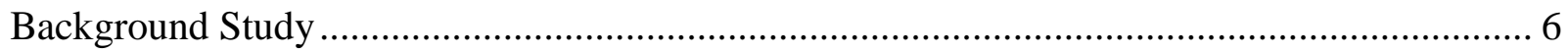

2.1 OCR

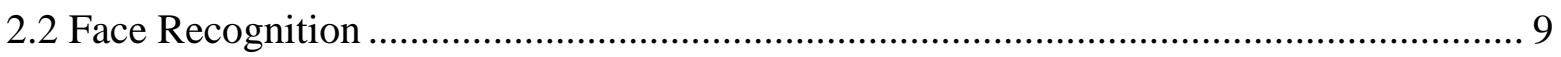

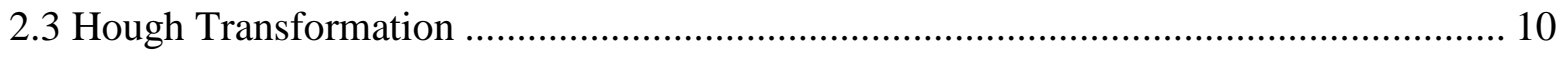

Proposed Methodology …………………………………............................................. 12

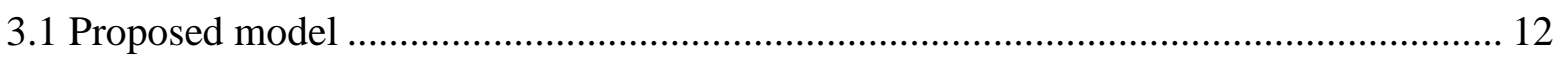

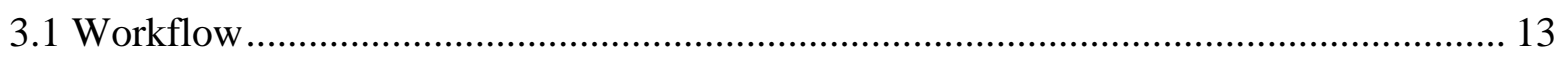

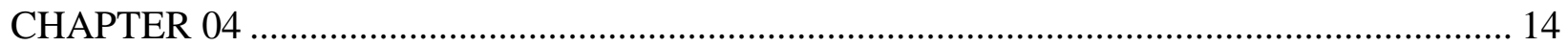

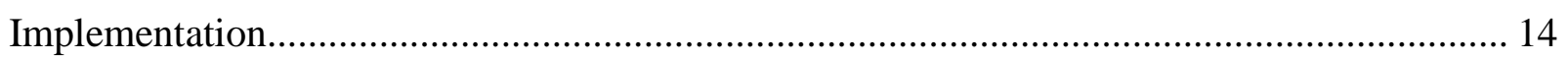

4.1 Required Tools and Programming Language ............................................................... 14

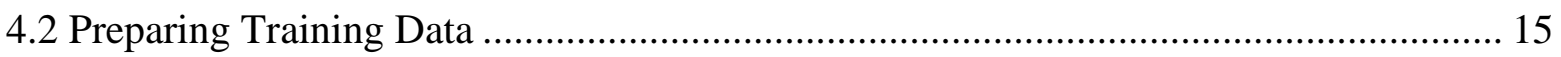

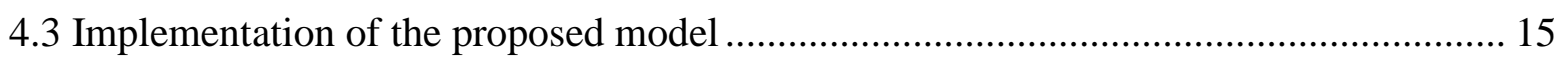

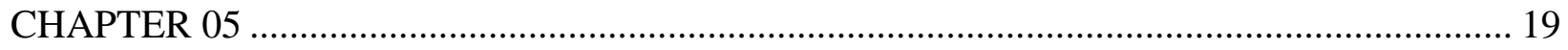

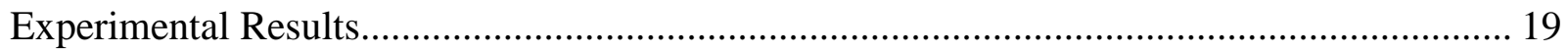

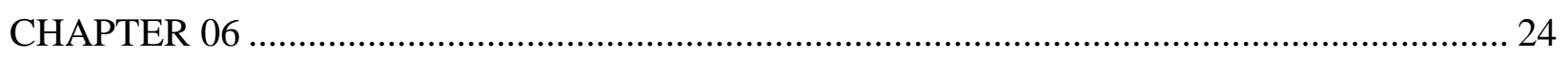

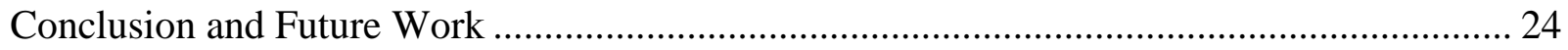

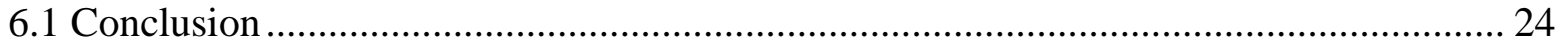

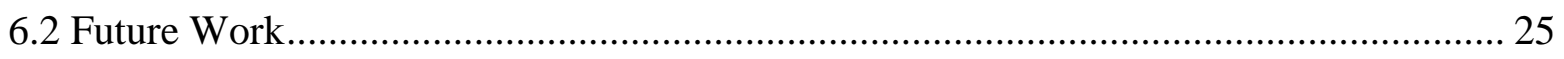

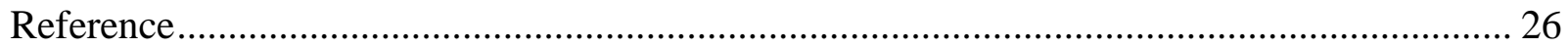

iii | P a g e 


\section{List of Figures}

Figure 1: Proposed Block Diagram.................................................................................. 12

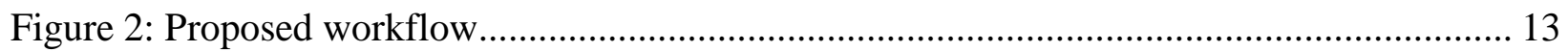

Figure 3: The input image to detect the feature of micro printing ............................................. 15

Figure 4: The input image to detect the feature of watermark...................................................... 16

Figure 5: The input image to detect the feature of UV lines .................................................... 16

Figure 6: Preprocessing image to detect the feature of OCR........................................................... 17

Figure 7: Gray scale to detect the feature of UV lines........................................................... 17

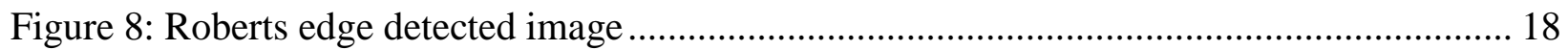

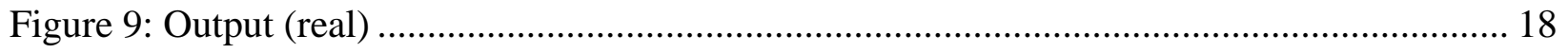

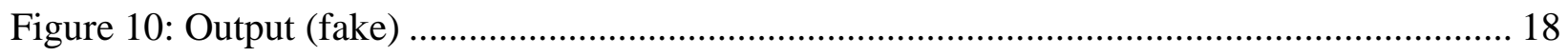

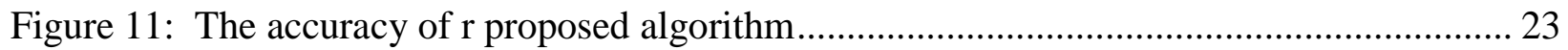

Figure 12: Run time of proposed algorithm graphically............................................................ 23

Figure 13: Percentage comparison of all the techniques ......................................................... 24 


\section{List of Tables}

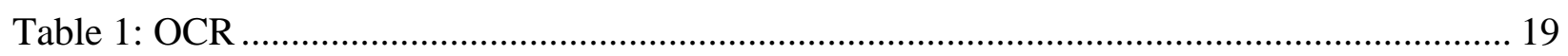

Table 2: Hough Transformation................................................................................... 20

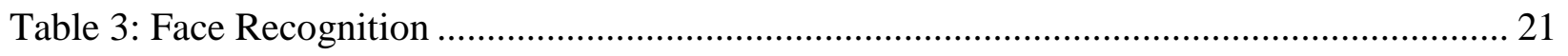

Table 4: Proposed model ................................................................................................ 22 


\section{List of Abbreviations}

\begin{tabular}{|c|l|}
\hline HSV & Hue Saturation Value \\
\hline OCR & Optical character recognition \\
\hline RGB & Red, Blue, Green \\
\hline RMS & Root Mean Square \\
\hline SD & Standard Deviation \\
\hline UV & Ultra Violet \\
\hline
\end{tabular}




\begin{abstract}
Currency duplication is now a common occurrence due to the advancement of printing and scanning technology. Many note detection systems are present in banks but they are very costly. In this paper, we are proposing an accurate and consistent technique for fake banknote recognition. We are developing an image processing algorithm which will extract different currency features and compare it with features of original note image. As an output, information about whether the note image is original or duplicate is given. Three main features of paper currencies has been implemented which are micro-printing, water-mark, and ultraviolet lines using OCR (Optical Character recognition), Face Recognition and Canny Edge \& Hough transformation algorithm of Matlab. We apply these techniques in order to find an algorithm which will easily be applicable and will be efficient in terms of cost, reliability and accuracy. Along with 1000taka note has been tested for checking authenticity hence making our techniques more appropriate for users.
\end{abstract}

Keywords: Digital Image processing, fake currency, counterfeit detection. 


\section{CHAPTER 01}

\section{Introduction}

Ever since the invention of currency, the concept of counterfeit currency has been around. Long before notes were used a form of money, counterfeiters would alter other forms of currency to gain more value than the traded item was worth. When the first coins were minted several thousand years ago, the value of the coin was based on the intrinsic value of the metal. Counterfeiters would scrape off small amounts of precious metal from real coins and use it to cover a cheap base metal and use it as a higher value coin. Ever since, counterfeit money has evolved into a huge black market. People may assume that counterfeit currency is not an issue anymore due to advancement in security features and detection technology, but it is actually the other way around. Nowadays, the cheap cost of inkjet printers has made it easier for more people to attempt counterfeiting, according to [1]. In [2], it was shown that every year, millions of fake notes are passed over retail counters and the majority is not identified as counterfeit until they're examined by the bank. A California resident was responsible for putting more than $\$ 7$ million in fake currency into circulation between 2004 and 2008, using ink jet and laser printers which he bought from a local store, according to [1]. He even checked them with counterfeit checking pen and found it was not being able to detect it as fake. There has been seemingly endless string of redesigns to US currency, hoping to stop the counterfeiters. However, the forgers fire back by reverse engineering those same features. In Bangladesh, similar occurrences have been observed. In 2016, 10 people were arrested along with a huge supply of counterfeit notes from different areas of Dhaka and Narayanganj. Counterfeit currency of worth 66 lacs, four laptops, and seven printers, two stacks of security threads used in 1,000 notes and a haul of fake currency making materials were detained from them, as mentioned in [3]. This year, in June, three persons of Belagavi, India were arrested for circulating fake Rs 2,000 notes which were found to be sourced from Bangladesh. These high quality forgeries, which are made with imported paper, look very much like genuine banknotes, contain watermarks and the long thread of banknotes, and are almost impossible to spot just by looking. The key to spotting them is to note if the color comes off when rubbed with wet fingers which is a tedious task in the case when there are a lot of notes involved. 


\subsection{Motivation}

Generally, people do not know how to identify legal money or they rely on the counterfeit pen, which is not a very reliable technology as applied in the design of the notes keep changing and the design of the pens need to be changed accordingly. Much research on the recognition of damaged bills has been conducted, and several methods based on different principles have been developed. Statistic parameters of existing methods are not sensitive to variation in the distribution of reflected light intensity as mentioned in [7]. Due to the variation of damages, the detection accuracy by existing technologies is still not satisfactory. Hence, detection of degree of damage remains an unsolved research subject. Automatic machines are available which can detect banknotes are now widely used in dispensers of modern products like candies, soft drinks bottle to bus or railway tickets, but are expensive and not handy for general citizens.

Basically, identifying and extracting visible and invisible features of currency notes is the main purpose of currency detection techniques. Till today, many techniques have been proposed to identify the currency note and the best way has been proven to use the visible features of the note i.e. the color and size of the note as mentioned in [17]. However, to make sure this also applies for torn and dirty money, it is important to properly and efficiently extract the features of the image of the currency note and apply proper algorithm to improve accuracy to recognize the note.

Digital image processing is the utilization of computer algorithms to apply those algorithms on digital images. Due to its versatile applications, digital image processing has many advantages over analog image processing. A wide range of algorithms can be applied to the input data and problems such as the build-up of noise and signal distortion during processing can be avoided. In [9], it is mentioned that digital image processing permits the use of much more intricate algorithms, and hence, can handle both complicated and simple tasks, and even implement the methods which would be impossible by analog means. Different algorithms are there such as Principal Component Analysis (PCA), Artificial Neural Networks (ANN) to Machine Visions to Support Vector Machines. All these give incredible results on finding counterfeit concerns.

Firstly, there are UV counterfeit detection scanners readily available in the market. It verifies the UV marks on authentic notes by shining UV (ultraviolet light) onto the bills. These UV marks 
are created while printing the money, through the use of apparently invisible dyes that are only visible under UV light. The bank note is expected to be authentic if the UV printed images glow when subjected to the UV light. There is similar currency detection machines which use other features of banknote to find its authenticity such as magnetic counterfeit detector, which uses magnetic detection to identify the magnetic ink and metal threads placed on strategic areas of bank notes. There are also watermark counterfeit detectors which use light to view watermarks that are built into authentic bank notes. On the other hand, size detection utilizes the fact that bills are all created in a standard size, so any deviation would indicate fake currency. Size detection adds an extra layer of protection. Lastly, automatic counterfeit detectors are the best way to accurately detect fraudulent bills. They are configured with advanced imaging software, allowing them to detect the exact dimensions and features of bank notes with $100 \%$ accuracy. They will automatically reject counterfeits. Among these, the ultraviolet counterfeit detection scanners are the cheapest and easily available in the markets of Bangladesh. However, since it only detects only one feature of the currency, we still need a better alternative. The counterfeit detector pen is a simple and cheap solution to counterfeit currency. It contains an iodine solution that reacts with the starch in wood-based paper to create a black stain. When the solution is applied to the fiber-based paper used in real bills, no discoloration occurs. If there is no starch present in the paper then the pen will indicate. The iodine in the pen reacts with starch that makes white paper look brighter. Most commercial paper, made from wood pulp, is brown unless bleached and starched. If the bill is authentic and the paper is fiber-based, there won't be any starch and the pen will not leave a mark.

The increased use of such devices has been motivated by progress in the fields of computerbased desktop publishing and scanning, which re security print technologies, such as intaglio an increasingly susceptible to counterfeit. One security documents against counterfeit is to print with optically variable devices which are replicated by scanners, and which can exhibit effects such as color changes by diffraction, and distinct switches between images. 


\subsection{Objectives}

- Detecting fake notes using OCR

- Detecting fake notes using Face Recognition

- Detecting fake notes using Hough Transformation

- Detecting fake notes using a revised algorithm for best results

- Tabulating and analyzing the difference in the results

\subsection{Contribution Summary}

- We achieved $93.33 \%$ accuracy for our proposed algorithm

- We developed our own dataset for face recognition part of our experiment

- We showed comparison between major image processing techniques

\subsection{Thesis Outline}

The thesis is organized as follows:

- Chapter 2 contains the background summary and an overview of the three main techniques

- Chapter 3 contains the block diagram and work flow of our works

- Chapter 4 shows the implemented work

- Chapter 5 shows the experimental results and tables

- Chapter 6 contains the future work and conclusion 


\section{CHAPTER 02}

\section{Background Study}

Many currency recognition machines are available in current market through which currency can be recognized. However, existing currency recognition systems are mainly based on image processing techniques like they did in [1, 2, 3, and 4]. At other cases, neural networks are implemented like done in $[21,22,23$, and 24]. There are also some other technologies used which are not as commonly used such as a counterfeit pen which is simply an inexpensive device that is designed to determine if a currency note is genuine or counterfeit, as mentioned in [16].

In [6], 'Currency Recognition on Mobile Phones', they adopted an approach based on computer vision on mobile devices, and develop an application that can run on low end smart phones. They have utilized the Open CV library for computer vision and image processing related tasks. While the camera interface is coded in Java, the language of Android, the image processing in the application is done in native $\mathrm{C}++$ code. The methods used work well on noisy images captured from a mobile phone. They faced some limitations in their system such as positioning of the camera might not be accurate which might lead to ambiguous results.

Similarly, in [5], 'Fake currency recognition using image processing and other standard methods' the limitation was that they did not perform rigorous testing to ensure the authenticity of the system, whereas in [1], they did not involve first line inspection methods in the algorithm which could potentially improve the performance of the system. On the other hand, in [4], they had some limitations relating to hardware and printing standard of Bangladeshi paper currency, as well as reported on improving the system with a micro controller based embedded software which would have support for All Kinds of Bangladeshi bank notes \& other foreign currencies. One of the main constraints of the system developed in [8] is the fact that the background of the image containing the banknote image must be contrasting with that object. Another constraint is that the illumination conditions over the image must be uniform. In [9], they lacked using cross validation method such as which can be used for currency recognition to make the system more reliable. They developed a good currency recognition system in [17], but their proposed technique was unable to distinguish genuine notes from counterfeits. 
In [14], 'Using Hidden Markov Models for Paper Currency Recognition' the test notes are placed on a system tray. Then, the system draws them successively and catches the images of definite sides of the banknote with a sensor. The algorithm is implemented with microcontroller; LPC-H2106.Upon performing the experiment, this application was able to determine the accurate values for $96.08 \%$ of the images tested. The system could not work properly in cases of dirty and torn notes. In [13], a machine based method is proposed. The micro printed portion of the notes is taken as the unique factor in this field. The micro printed text on those notes is "BANGLADESHBANK". The method is efficient but time consuming. Another drawback for this method is the positioning of the grid scanner. The system reports excellently when the scanner positioning is flawless. However, the inclination rate of the scanner results in an incorrect identification product.

A Radial Basis Function Network for classification is used in [23]. Saudi Arabian paper currency is used in this case as a model. In building such a structure, diverse dimensions, areas, Euler numbers and correlations as features are all checked profoundly. The objective of the paper is to accomplish the best precision in spotting designs with the discounted cost possible. In radial basis neural networks, there are three layers: input layer, hidden layer and output layer. The input layer contains the construction or configuration of the image which are considered as data. The hidden layer is used to transform the image into non-linear transformation from input layer space to hidden layer space. The technique requires high number of neurons. The output layer does the opposite. It transforms the image's non-liner form and converts it to linear form. The radial basis function network classifier was verified with a database of 110 images, 10 of which are tilted with an angle less than $150.91 .51 \%$ was seen as average acknowledgement rate. The masses of the connections between neurons works as a negative factor towards congregate the best values. The mentioned error eradication can make the method more sophisticated in detecting fake notes. An unlimited-vocabulary OCR system for English and Arabic was proposed in [24]. They based their system on Hidden Markov Models (HMM). Recently, this approach has been widely accepted in the area of automatic speech recognition. They showed character recognition with unlimited vocabulary using word-based HMM system to perform. Using trigram language model on character sequences techniques, they achieved very low error rate. They showed how the HMM conditional independence assumption leads to less than best possible recognition accuracy 
on a natural mix of data from different styles is used for training. They did not test their system on noisy data, which remains a limitation of their project.

For face recognition, in $[3,11,15,19]$ it was shown that a computer program provided with face features extracted manually could perform recognition with good performance. It is shown that face can be recognized even when the details of the individual features cannot be resolved. The remaining information is completely geometrical, thus extracting relative position and other parameters of distinctive features such as eyes, mouth, nose, and chin. In [13] and [2], a more recent approach is used. In [40], a correlation-based approach was made for face recognition. They followed two approaches which are identification through a vector of geometrical features and identification through a template matching strategy. They did not test it on larger database and did not contain reliable rejection of images of face. Moreover, they could have expanded their set of templates which they did not. In [4], they have constructed a face detection algorithm for color images in the presence of varying lighting conditions and complex backgrounds. They have received good results over a wide variety of facial variations in color, position, scale, rotation, pose, and expression from many dataset. However, they did not consider distorted or blurred images.

In [20], 'A basic study on human face recognition' line detection using Hough transformation has been performed as well as discussion about cluster detection in Hough space has been conducted. They received mixed results including some good results and some errors. Their system could not give an accurate result for unbalanced lines which is a limitation. More dataset training could improve their system.

Ultimately, image processing is the emerging field where our topic in hand is going to embark on; hence, here we have discussed some commonly applied image processing techniques.

\subsection{OCR}

Optical character recognition (OCR) is process of classification of optical patterns contained in a digital image corresponding to alphanumeric or other characters. The character recognition is achieved through important steps of segmentation, feature extraction and classification [12]. 
OCR has gained increasing attention in both academic research and in industry. Over the last few decades machine reading has grown from dream to reality through the development of sophisticated and robust Optical Character Recognition (OCR) systems. OCR technology enables us to convert different types of documents such as scanned paper documents, PDF files or images captured by a digital camera into editable and searchable data. OCR systems have become one of the most successful applications of technology in pattern recognition and artificial intelligence field. The main concept in automatic recognition of patterns is first to teach the machine which class of patterns that may occur and what they look like [3,4]. OCR patterns are letters, numbers and some special symbols like commas, question marks as well as different characters. The teaching of machine is performed by showing machine examples of characters of all different classes. Based on these examples the machine builds prototype or description of each class of characters. During recognition the unknown characters are compared to previously obtained descriptions and assigned to class that gives the best match. In most commercial systems for character recognition training process is performed in advance. MATLAB has built in OCR system in it. We will use that system.

\subsection{Face Recognition}

Facial recognition systems utilizes an algorithm that can predict that face match based on many points on an individual's face, given a set of dataset. It measures the overall facial structure, including distances between eyes, nose, mouth, and jaw edges. These measurements are retained in a database and used as a comparison when a user stands before the camera. Capturing, extracting, comparing, matching are the four core stages of face recognition. Face recognition is one of the highest levels of security since it only works by the face of people which is not possible to imitate or hide. Software is being developed for computer networks and automated bank tellers that use facial recognition for user verification purposes. In case of image processing, face recognition uses computer vision to extract information from facial images, and pattern recognition or machine learning techniques to model the appearance of faces and to categorize them. MATLAB and openCV are common platforms to perform such applications. We considered PCA and MSD for our work and ultimately decided to choose MSD because the MSD method is more efficient than PCA and the rate of success of MSD method is better than PCA. Moreover, the MSD method is faster because PCA involves calculating the eigenvectors of 
a big covariance matrix. In image processing, mean is used for noise reduction in images. Mathematically, mean is shown as,

$$
\mu=\frac{\sum_{i=1}^{n} x_{i}}{n}
$$

In image processing, standard deviation is used in edge sharpening, as intensity level get changes at the edge of image by large value as it has ability to measure the variability. Standard deviation displays how much variation occurs at mean value. A low standard deviation states that the data points tend to be very close to the mean, where high standard deviation specifies that the data points are spread out over a large range of values. Mathematically, standard deviation is a measure of how spread out the data set from the mean.

$$
\sigma=\sqrt{\frac{\sum_{i=1}^{n}\left(x_{i}-\mu\right)^{2}}{n}}
$$

\subsection{Hough Transformation}

The Hough transform is a technique which can be used to isolate features of a particular shape within an image. The Hough transform can be used to identify the parameter of a curve which best fits a set of given edge points. This edge description is commonly obtained from a feature detecting operator such as the Roberts, Sobel or Canny edge detector and may be noisy, i.e. it may contain multiple edge fragments corresponding to a single whole feature. Furthermore, as the output of an edge detector defines only where features are in an image, the work of the Hough transform is to determine both what the features are (i.e. to detect the feature for which it has a parametric (or other) description) and how many of them exist in the image. The classical Hough transform is most commonly used for the detection of regular curves such as lines, circles, ellipses, etc. in our case, we are considering detection of lines, and hence classical Hough transformation should be enough for us. Despite its domain restrictions, the classical Hough transform retains many applications; as most manufactured parts contain feature boundaries which can be described by regular curves. The main advantage of the Hough transform technique is that it is tolerant of gaps in feature boundary descriptions and is relatively unaffected by image 
noise. Also, they are conceptually simple, easily implemented, handles missing and occluded data gracefully and can be adapted to many types of forms, not just lines.

The representation of straight lines is the polar (also called normal) representation of straight lines is

$$
x \cos \theta+y \sin \theta=\rho
$$




\section{CHAPTER 03}

\section{Proposed Methodology}

\subsection{Proposed model}

Figure 1 demonstrates the block diagram that represents the implementation procedure of the proposed model. It shows the block diagram of the proposed algorithm. In the proposed algorithm at first collect the data which we will input in MATLAB as a JPEG image. Then we will preprocess the images to get the desired mode of picture for feature extraction. Then, for more accuracy, we will perform edge detection. After that, the algorithm will go through feature extraction and ultimately identification of the note as fake or real.

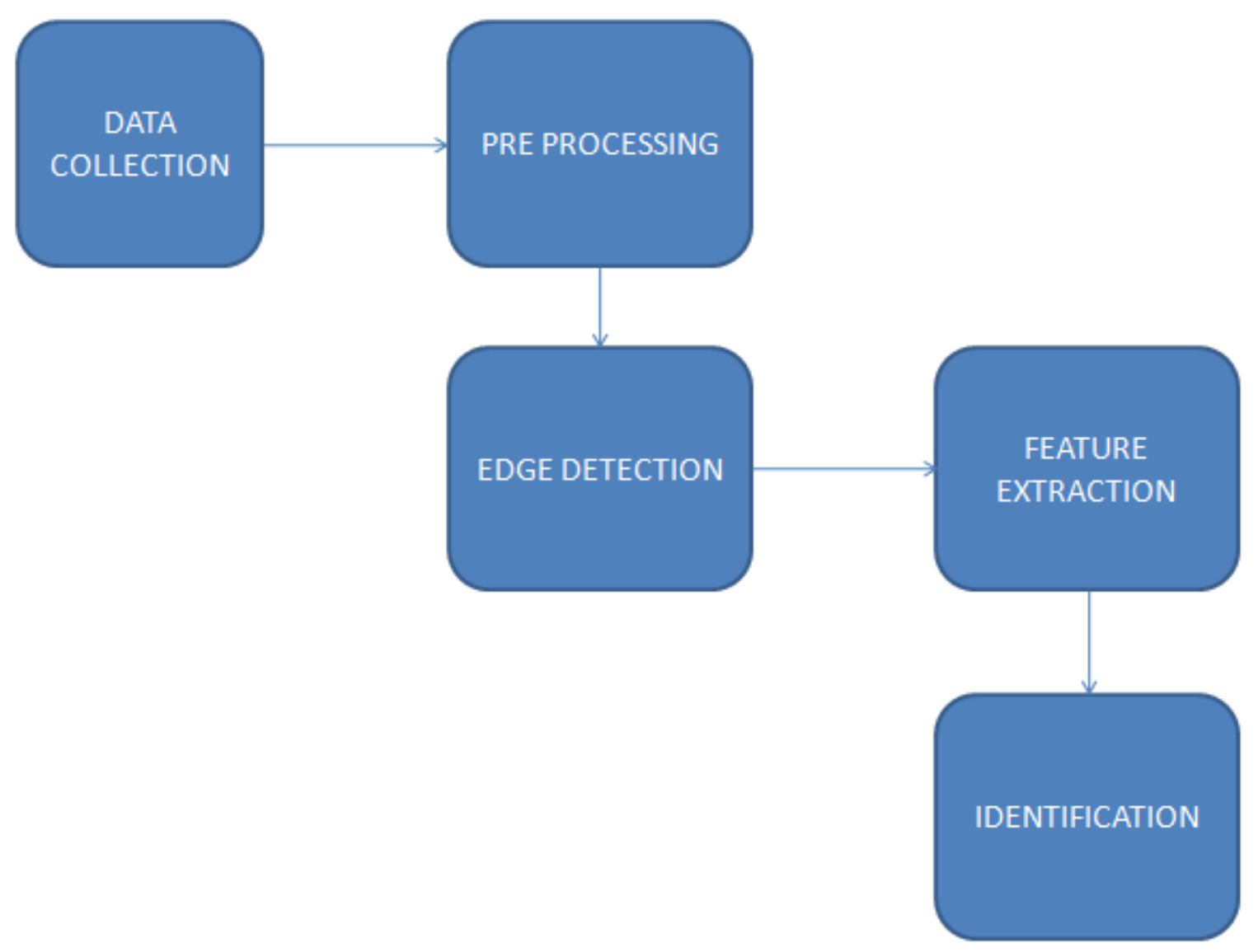

Figure 1: Proposed Block Diagram 


\subsection{Workflow}

Figure 2 shows the work flow diagram of the proposed model. At first, the input image goes thorough OCR and upon successful recognition of OCR, the variable " $\mathrm{fc}$ " which stands for feature counts, is made $\mathrm{fc}++$. It is to mention that at the beginning of the code, fc is set as 1 and goes higher from there. Then, the algorithm of face recognition is used and upon successful recognition, fc++ is used again. Similarly, the input image goes through Hough transformation algorithm for UV lines detection and finally the fc value determines the authenticity of the note. If the value is greater than 1 , the note is considered real. If any of the features are successfully extracted, the note should be real because these are the strongest features of notes.

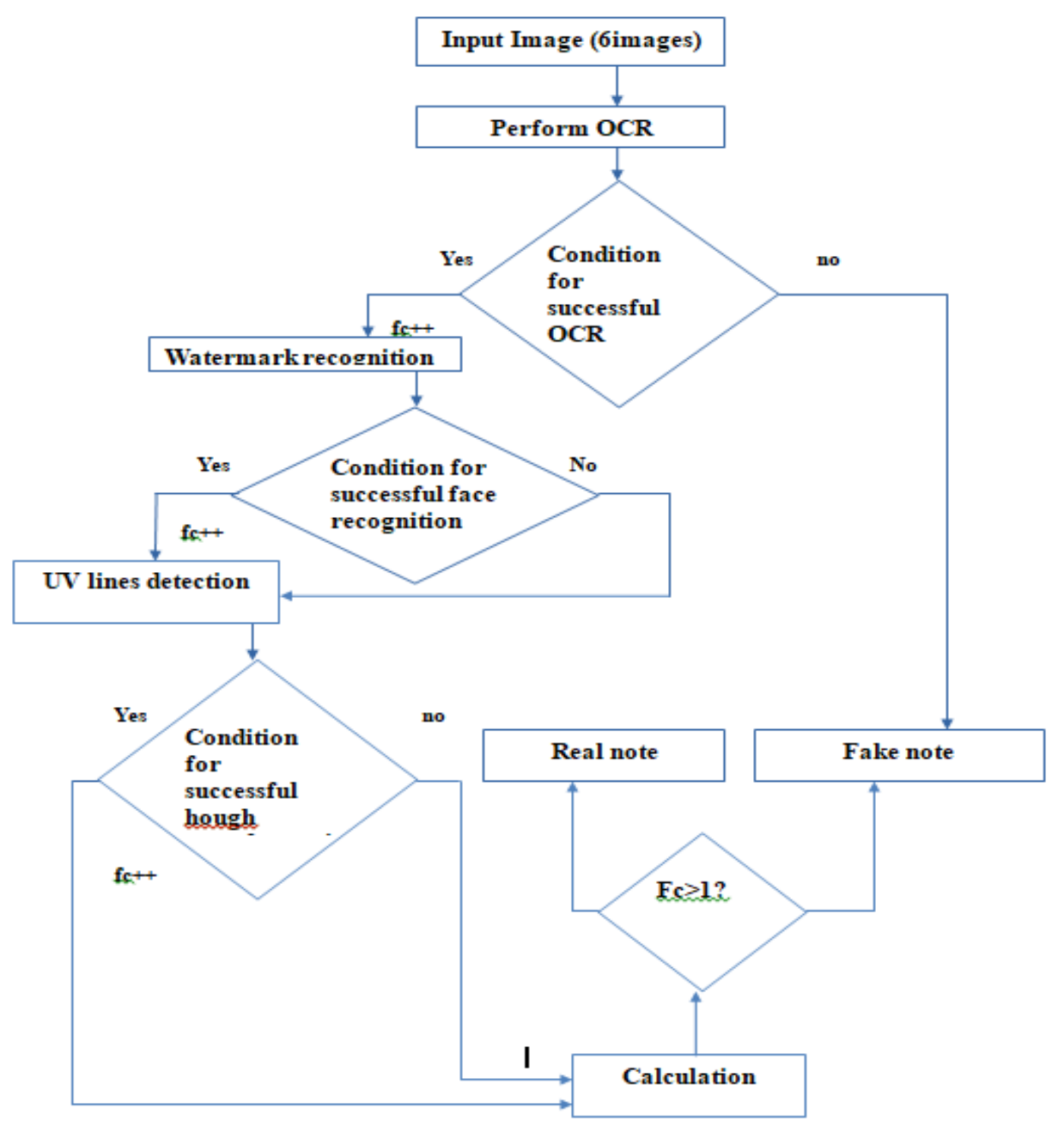

Figure 2: Proposed workflow 


\section{CHAPTER 04}

\section{Implementation}

The tasks that we are undergoing the recognition of fake notes are listed below:

- Preparing Training data for face recognition

- Pre-processing three different types of input images

- Perform edge detection for specific algorithm

- Performing Recognition using three different algorithms

- Showing the output result

\subsection{Required Tools and Programming Language}

Here is a list of required tools and Programs

- MATLAB

- 16megapixel camera

- UV light

- Macro lenses

- We have implemented our algorithm in MATLAB (version8.4.0 (64bit). All the experiments were performed on a computer bearing the configuration as follows:

$>$ CPU: Intel Core i3-5010U CPU $2.10 \mathrm{GHz}$

$>$ RAM: 4 GB DDR3 1333MHz

$>$ Operating System: Windows 10 64bit. OCR was the technique applied.

We preferred MATLAB over openCV as the results are expected to be more accurate. UV lights are used to detect lines of real banknotes. Macro lenses are used to detect micro letters from the notes which are not visible to bare eyes. Finally, we have implemented our algorithm in the strongest configuration laptop to get the best results.

\subsection{Preparing Training Data}

In our proposed model, initially, we will collect our data. So we to first accumulate real bank note in order to run our training tests. Hence, we used 20 of newly printed 1000taka notes. Then, we had to collect fake notes of the same value from bank with the help of an authorization letter from our supervisor, in order to run tests on our already developed algorithm and training set. 
Then, we used camera to collect all the pictures to apply them on MATLAB. This part is the image acquisition part.

The features of banknote that we are considering are watermarks of The Portrait of Bangabandhu Sheikh Muzibur Rahman, microprints of the texts 'BANGLADES H BANK' and Ultraviolet lines which can only be seen through UV light.

After obtaining the image in our desired format, we run our test in MATLAB. Firstly, we have done image acquisition part where we take a picture of the note and then we read that image with the help of MATLAB.

\subsection{Implementation of the proposed model}

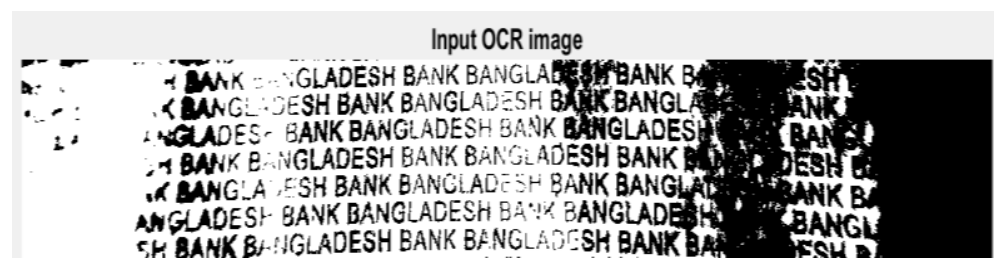

Figure 3: the input image to detect the feature of micro printing

Figure 3 shows the input image to detect the feature of micro printing which is basically image acquisition. We plan to take picture of 1000taka note using 16megapixel camera of a smartphone. Our test set will contain 30input images and each will be tested for accuracy. We used additional macro lenses to obtain such images because the micro prints cannot be detected using normal camera. In the case of fake notes, there will not be any micro letter detected as that technology has not been used as forgery as yet. 


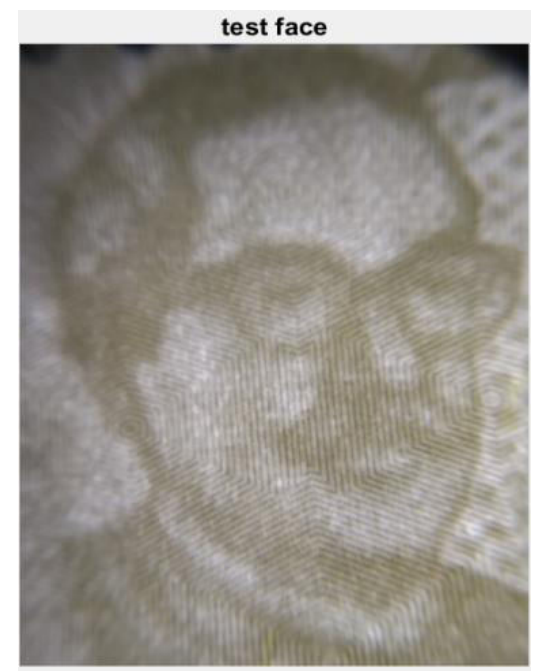

Figure 4: the input image to detect the feature of watermark

Figure 4 shows the input image of Face recognition for our proposed algorithm for the similar image acquisition part. For the face recognition part, we will have to go through an additional training image part beforehand. In that part, 5 images of Bangabandhu will be trained and stored in database of MATLAB. Then, after the test face is given, it will compare that picture after extracting the mean standard deviation features. Finally, it will give us the result.

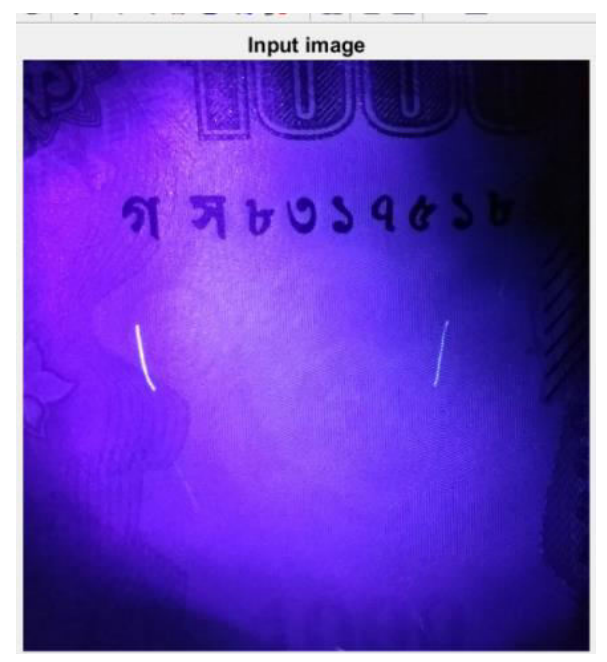

Figure 5: the input image to detect the feature of UV lines

Figure 5 shows the input image for extracting UV security lines. We used an additional UV light to capture such images. For the fake image, no lines of UV were detected, whereas for real notes, there were many security lines observed. 


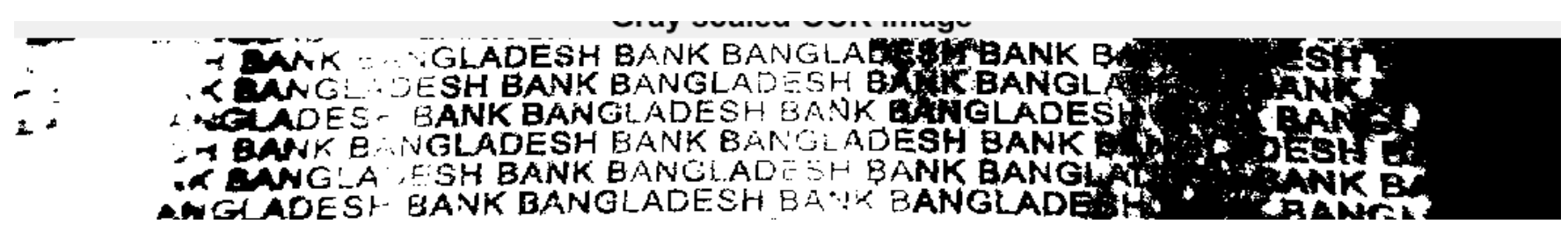

Figure 6: preprocessing image to detect the feature of OCR

Figure 6 shows the preprocessing part of the OCR proposed algorithm which performs de noising, rotating and gray scaling for applying the feature extraction.

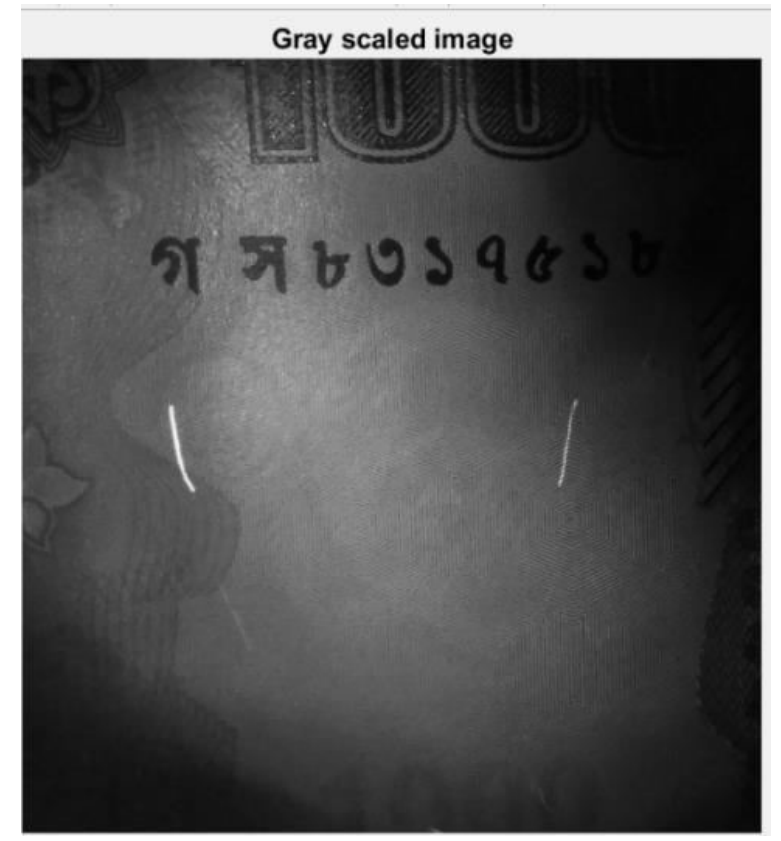

Figure 7: Gray scale to detect the feature of UV lines

Figure 7 shows the preprocessing part of the Hough transformation part of our algorithm which performs gray scale to recognize the UV lines better. 


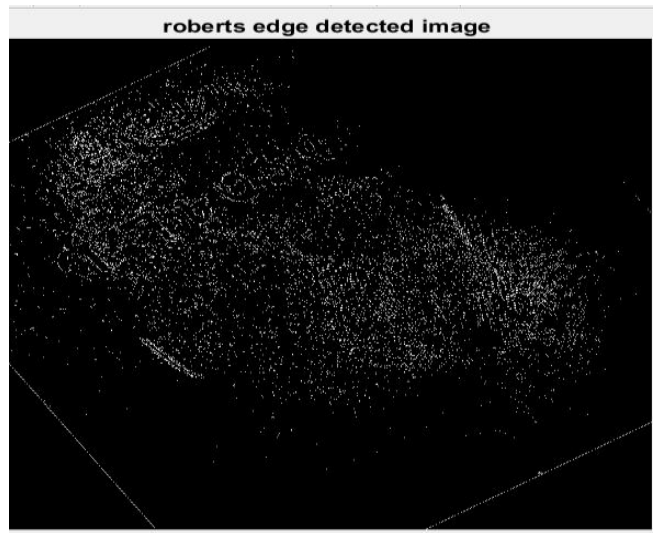

Figure 8: Roberts edge detected image

Figure 8 shows the edge detection part. Here we will apply Roberts edge detection in order to detect the lines better. We need edge detection only in the case of UV lines detection since the lines can be anywhere in the note. Hence, proper edge detection will make it easier to detect the lines.

After this, the algorithm goes through checking the variable that was set for the number of correct outputs of notes. After calculating the values, the output will be shows as real or fake as follows

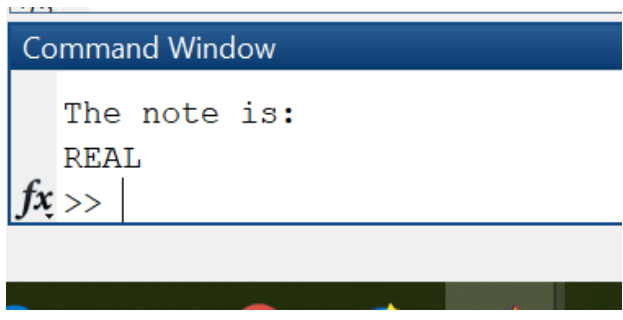

Figure 9: Output (real)

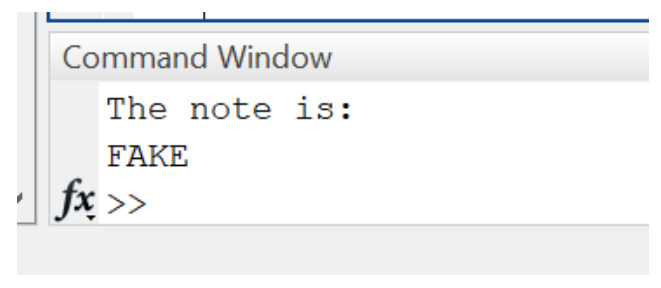

Figure 10: Output (fake) 


\section{CHAPTER 05}

\section{Experimental Results}

Table 1: OCR

\begin{tabular}{|c|c|c|}
\hline $\begin{array}{l}\text { AD(Accurate } \\
\text { detection) }\end{array}$ & Number & Run times(seconds) \\
\hline Yes & 12 & $\begin{array}{c}3.92,5.46,3.15,3.05,3.05,3.48 \\
3.46,4.63 .1 .90,2.89,3.23,3.61\end{array}$ \\
\hline No & 8 & $\begin{array}{c}\text { 3.15, 3.22,3.29, } 3.52,1.79,2.10, \\
3.20,3.03\end{array}$ \\
\hline
\end{tabular}

Table 1 shows the results of when we only applied OCR algorithm to the notes. We considered fresh and new notes in most cases. Even then, it was hard to detect the micro letterings accurately since the printings were extremely small. Overall it is the most credible way of checking authenticity of a note but may not always work based on the quality of the input image.

Accuracy rate $=($ No. of Correct readings/Total No. of readings $) * 100=(27 / 30) * 100=73.33 \%$

Average time for code to run $=3.6065$ seconds

Table 2: Hough Transformation

\begin{tabular}{|c|c|c|}
\hline $\begin{array}{c}\mathrm{AD} \text { (Accurate } \\
\text { detection) }\end{array}$ & Number & Run times(seconds) \\
\hline Yes & 15 & $\begin{array}{c}\text { 3.98,4.12, 3.56, 6.19, 4.33, 3.67 } \\
\text { 3.98, 3.41, 3.66, 5.78, 4.97 } \\
4.16,4.88,3.76,4.51\end{array}$ \\
\hline No & 5 & $3.33,4.56,3.66,3.92,4.67$ \\
\hline
\end{tabular}

Table 2 shows the results of when we only applied Hough transformation algorithm to the notes. The detection of lines was complex because arbitrary lines are put in notes as security lines. Hence, different notes had different results in terms of run time and detection accuracy. Overall, this technique was more accurate than OCR but was more time consuming than OCR.

Accuracy rate $=($ No. of Correct readings/Total No. of readings $) * 100=(24 / 30) * 100=80 \%$

Average time for code to run $=4.285714$ seconds

Table 3: Face Recognition 


\begin{tabular}{|l|l|c|}
\hline $\begin{array}{c}\text { AD(Accurate } \\
\text { detection) }\end{array}$ & \multicolumn{1}{|c|}{ Number } & Run times(seconds) \\
\hline Yes & 12 & $\mathbf{5 . 1 2}, \mathbf{5 . 8 7}, \mathbf{4 . 9 1 , 4 . 5 5 , 5 . 4 3 ,}$ \\
& & $\mathbf{5 . 1 1}, \mathbf{5 . 7 2}, \mathbf{5 . 9 9 , 5 . 5 2 , 4 . 3 7 ,}$ \\
& & $\mathbf{5 . 4 4 , 4 . 8 3}$ \\
\hline No & 8 & $\mathbf{5 . 5 6 , 4 . 5 6 , 5 . 9 7 , 4 . 7 0 , 4 . 1 2 ,}$ \\
& & $\mathbf{5 . 4 9 , 5 . 7 1 , 4 . 5 1}$ \\
\hline
\end{tabular}

Table 3 shows the results of when we only applied face recognition algorithm to the notes. Face recognition had very low accuracy and run time was also slower.

Accuracy rate $=($ No. of Correct readings/Total No. of readings $) * 100=(27 / 30) * 100=76.67 \%$ Average time for code to run $=5.374$ seconds

Table 4: Proposed model

\begin{tabular}{|l|l|c|}
\hline $\begin{array}{c}\text { AD(Accurate } \\
\text { detection) }\end{array}$ & Number & Run times(seconds) \\
\hline Yes & 18 & $\mathbf{6 . 1 3 , 6 . 6 6 , 6 . 9 0 , 6 . 5 6 , 6 . 1 6 ,}$ \\
& & $\mathbf{6 . 7 2 , 6 . 6 4 , 6 . 9 1 , 7 . 0 1 , 6 . 6 5 , 6 . 3 9 ,}$ \\
& & $\mathbf{6 . 2 2}, \mathbf{6 . 9 3}, \mathbf{6 . 4 2}, \mathbf{6 . 9 4 , 6 . 9 8 , 6 . 0 4 ,}$ \\
\hline No & 2 & $\mathbf{6 . 3 3}$ \\
\hline
\end{tabular}

Table 4 shows the results of proposed algorithm where we applied all the above techniques and obtained optimum results. The run time was not very largely increased while the accuracy was raised up to $93.33 \%$.

Accuracy rate $=($ No. of Correct readings/Total No. of readings $) * 100=(27 / 30) * 100=93.33 \%$ Average time for code to run=6.1895 seconds 


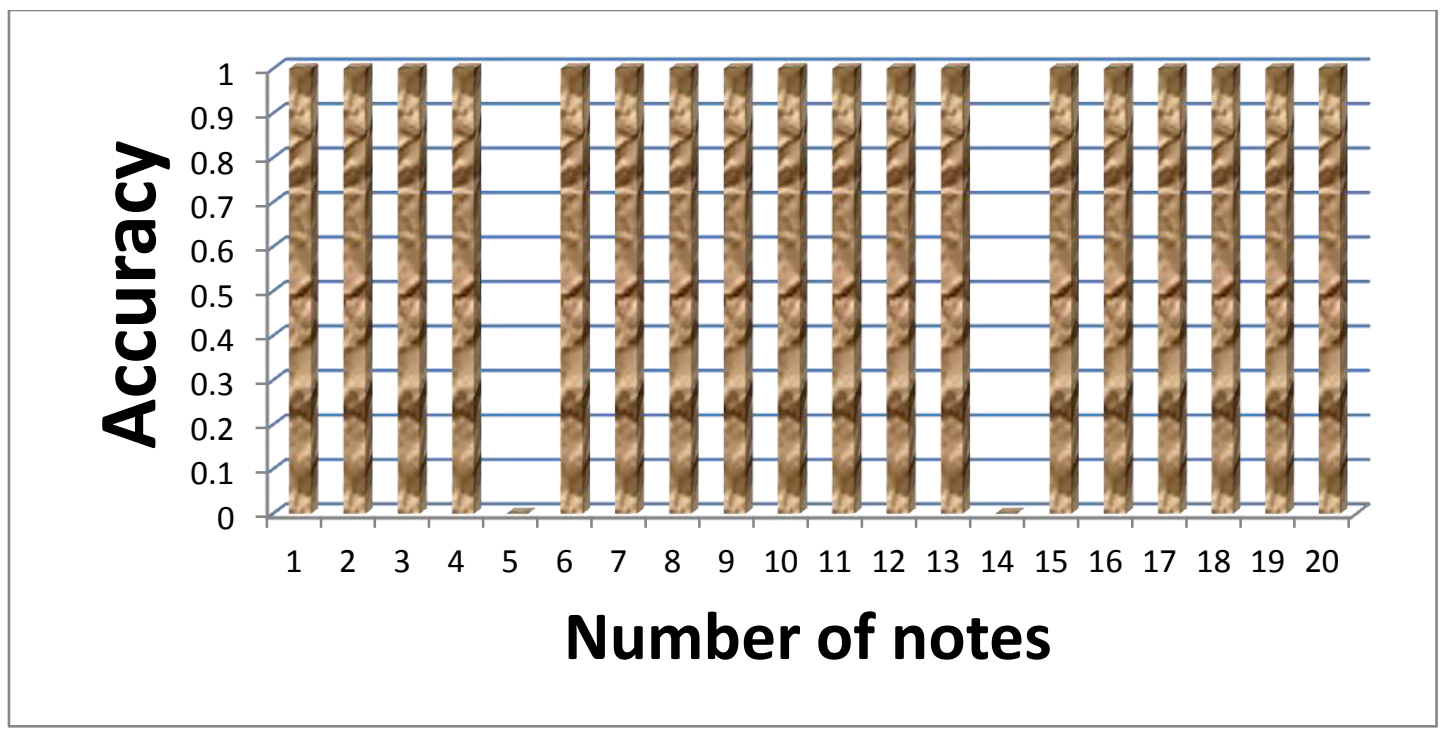

Figure 11: the accuracy of proposed algorithm

Figure 11 shows the accuracy of our proposed algorithm and it is evident that almost in every case, correct detection has been performed. Therefore, our proposed system was successful.

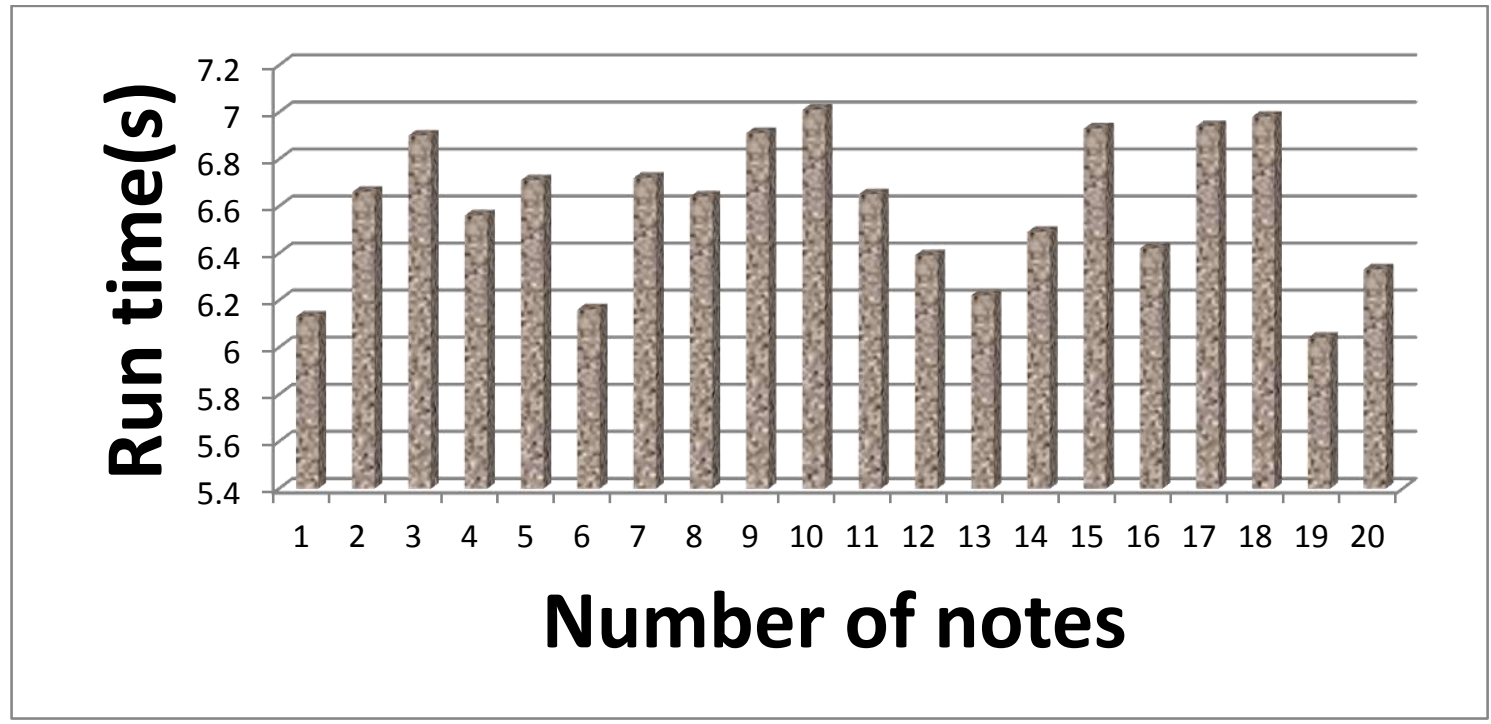

Figure 12: run time of proposed algorithm graphically

Figure 12 shows the run time of our proposed algorithm which shows the average time needed for the code to run. Overall the highest time needed has not exceeded 7 seconds and the lowest time needed was 6 seconds. 


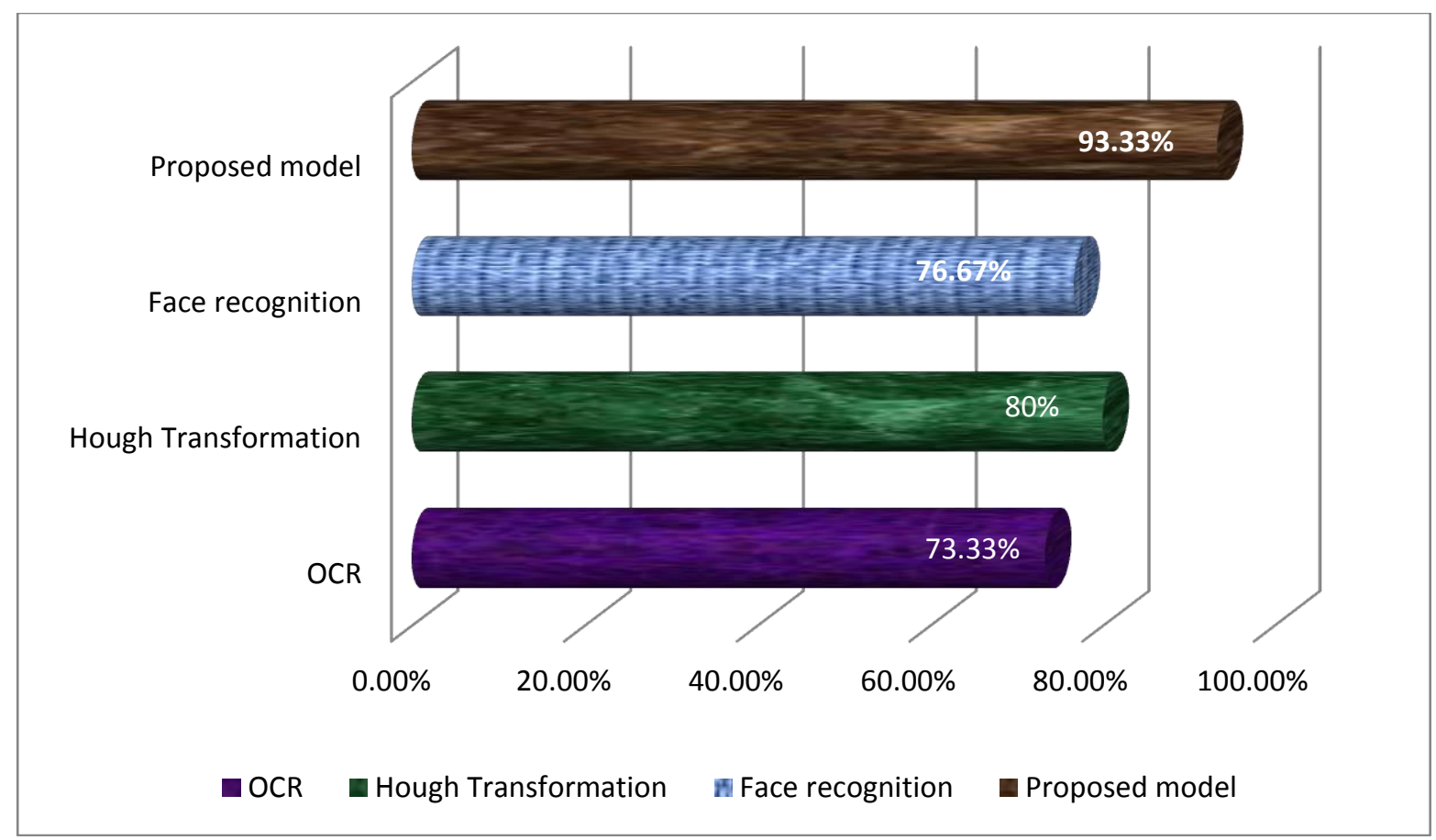

Figure 13: percentage comparison of all the techniques

Figure 13 shows overall comparison between the accuracy of our proposed algorithm and the individual algorithms. It should be mentioned here that we conducted our own experiment to attain the results of the individual algorithms as well as our proposed algorithms. Therefore, we did not have to refer to any previous work to show the comparison. It shows that OCR has obtained the lowest accuracy while the other two techniques had $76.67 \%$ and $80 \%$ accuracy. Our proposed algorithm has highest accuracy.

After implementing each of our intended algorithms, we inferred some outcomes. We implemented each of the algorithms individually, and then combined the algorithms in order to observe any change.

First of all, we implemented OCR which gave us accuracy of $73.33 \%$. However, relying on only one technique may be risky as new technologies are emerging constantly and new techniques of micro printing on fake notes may come around in future.

The result of face recognition was more accurate than that of OCR it might be due to the fact that the micro letterings get faded out easily hence give inaccurate results at times. 
Hough transformation took the shortest time to run. However, due to many other writings and symbols all over the note, in some cases, the algorithm detected lines which are not actually part of the security UV lines. Hence, the system showed better performance when OCR and face recognition were applied together. Overall, when the algorithms were combined, they showed better accuracy but took longer to run. On the other hand, more than one technique was preferable to be used in order to make sure that the fake notes were not being recognized as real.

Lastly, we combined the face recognition, OCR and Hough transformation algorithm and this gave better accuracy than all the other ones. This ensured more reliable results in terms of detecting fake notes. However, this algorithm took the longest to run due to the accumulation of all the algorithms at one go. Nevertheless, it gave us the highest accuracy of $93.33 \%$ which was the main motive of our work. 


\section{CHAPTER 06}

\section{Conclusion and Future Work}

\subsection{Conclusion}

Fake currency production without the legal sanction of the government is an increasing threat to Bangladesh at present. Recently, counterfeit of 10taka note has also started to prevail due to the technological advancement and due to lack of awareness. Some of the effects that counterfeit money has on society include a reduction in the value of real money, inflation due to more money getting circulated in the society or economy which in turn dampen our economy and growth - an unauthorized artificial increase in the money supply, a decrease in the acceptability of paper money and losses. This has led to the increase of corruption in our country and thus hindering economic growth of Bangladesh.

Moreover, since there has been significant technological development in the field of banking services, automatic methods for paper currency recognition are important in many applications around us. Therefore, the right kind of technological investments are needed to be made in order to find and root out money counterfeiting networks on a large scale. Furthermore, global experts can be consulted about putting more security features into new banknotes in the long term. In the meantime, every citizen should stay vigilant against this growing menace. In order to be able to do so, image processing techniques can come in handy to easily and quickly detect counterfeits. In this project, our fake currency detection technique using MATLAB is expected to overcome such drawbacks as well as contribute in mitigating such forgery altogether.

In our thesis work, we have successfully proposed an algorithm that achieved $93.33 \%$ accuracy for our proposed algorithm. We developed our own dataset for face recognition part of our experiment. Moreover, we showed comparison between major image processing techniques which will help future improvements in the field of fake currency recognition. 


\subsection{Future Work}

We have not considered torn and dirty notes which can be a future expansion of our work. However, that will be a very complex implementation since the micro printings become faded out very easily due to being passed to many users consecutively. In that case, the main algorithm for us, the OCR algorithm, will be very hard to implement.

We excluded security thread and optical variable ink from our consideration, but we could implement it in the future to test if more accuracy is obtained. If we do so, the process may be slower and inefficient since we will need perfect pictures of security thread and many angle pictures of optical variable ink. Also, that algorithm may not be appropriate for general citizen as they will look for instant outputs from the fake note detection tests. We plan to implement a machine learning technique to solve that issue, preferably the SVM (Support Vector Machine) technique in order to achieve an optimum algorithm. It should be mentioned here that, large amount of dataset will be required in order to apply that process. Moreover, 10taka notes forgery are very common these days, we could work with those too, in order to increase efficiency of our system, as well as combating forgery in a stronger way. 


\section{Reference}

[1] H. Hassanpour, A. Yaseri, G. Ardeshiri, Feature Extraction for Paper Currency Recognition,IEEE, 2007.

[2]Zahid Ahmed, Sabina Yasmin, MdNahidul Islam, RaihanUddin Ahmed, Image Processing Based Feature Extraction of Bangladeshi Banknotes, 978-1-4799-6399-7/14 ed. Bangladesh: IEEE, 2014.

[3] Mohammad Shorif Uddin, Pronaya Prosun Das, Md. Shamim Ahmed Roney, Image-Based Approach for the Detection of Counterfeit Banknotes of Bangladesh, 2016 5th International Conference on Informatics, Electronics and Vision (ICIEV) ed. Japan: International Conference on Informatics, Electronics and Vision (ICIEV)

[4] Prof. Sagar S. Rajebhosale, Devang S. Gujarathi, Sushil V. Nikam, Currency Recognition System Using Image Processing, Volume 4, Issue 3 ed. India: International Research Journal of Engineering and Technology, May 2017.

[5] D. Alekhya, G.Devi Surya Prabha, G. VenkataDurgaRao, fake currency recognition using

image processing and other standard methods, volume 3, issue 1 ed. India: International Journal of Research in Computer and Communication Technology, January 2014.

[6]Suriya Singh1 Shushman Choudhury2 1CVIT, IIIT Hyderabad, India Kumar Vishal1 2 IIT Kharagpur, India C.V. Jawahar1, Currency Recognition on Mobile Phones, 22nd ed. China: International conference on pattern recognition , 2014.

[7]Ballado, A. H. Jr., Avdendano, G. O.,Echano, Philippine Currency Paper Bill Counterfeit Detection Through Image Processing Using Canny Edge Technology , 978-1-5090-0360-0 ed. Manila, Philippines: IEEE, 2015.

[8]Felipe Grijalva, J. C. Rodriguez, Julio Larco, Luis Orozco, Smartphone Recognition of US Banknotes Denomination for Visually Impaired People, 978-1-4244-6742-4 ed. Ecuador: IEEE, 2010. 
[9]Vishnu R, BiniOmman, Principle Features for Indian Currency Recognition, 978-1-47995364-6 ed. india: IEEE, 2014.

[10]Binod Prasad Yadav, C. S. Patil, R. R. Karhe, P. H. Patil, An Automatic Recognition of Fake Indian Paper Currency Note Using Matlab, Volume 3, Issue 4 ed. india: International Journal of Engineering Science and Innovative Technology, July 2014.

[11]M. Deborah, C. SoniyaPrathap, M. E., Detection of Fake Currency Using Image Processing, Volume 1, Issue 10 ed. India: International Journal of Innovative Science, Engineering and Technology, December 2014.

[12] P. Ponishjino, Kennet Antony, Sathish Kumar, SyamJeba Kumar, Bogus Currency Authorization Using HSV Techniques, 978-5090-5686-6 ed. India: International Conference on Electronics, Communication and Aerospace Technology, 2017.

[13]Hasanuzzaman F.M, Xiaodong Yang, YingLiTian, Robust and EffectiveComponent-Based Banknote Recognition for the Blind, Systems, Man,and Cybernetics, Part C: Applications and Reviews, IEEE Transactionson, vol.42, no.6, pp.1021,1030, Nov 2012.

[14]Hamid Hassanpour, Payam M, Farahabadi, Using Hidden Markov Modelsfor Paper Currency Recognition, In Journal of Expert Systems withApplications, Vol. 36, No. 6, pp. 10105-10111, 2009.

[15]Liu, J. and B Kessel (2008).A New Image Processing Method for Damaged Bill Recognition, IEEEJournal, pp. 495-498.

[16] Sajal, R.F., Kamruzzaman, M. and Jewel, F.A., 2008, December. A machine vision based automatic system for real time recognition and sorting of Bangladeshi bank notes. In Computer and Information Technology, 2008.ICCIT 2008. 11th International Conference on (pp. 533-535). IEEE. 
[17]ChinmayBhurkey, MeghanaSirdeshmukh, Prof Mrs. M.S. Kanitkar, currency recognition using image processing, volume 3, issue 5 ed. India: International Journal of Innovative Research in Computer and Communication Engineering, May 2015.

[18] A. J. Goldstein, L. D. Harmon, and A. B. Lesk, "Identification of humanfaces," Proc. IEEE,,vol. 59, p. 748, 1971.Some preliminary results,” Tech. Rep., Panoramic Res. Inc., Palo Alto,CA, 1965.

[19] 2018. [Online]. Available: https://www.thedailystar.net/city/6-held-tk-66-lakh-fake-notesdhaka-1304575.

[20] Y. Kaya and K. Kobayashi, "A basic study on human face recognition,"in Frontiers of Pattern Recognition (S. Watanabe, Ed.).1972, p. 265.

[21] G. Cottrell and M. Fleming, "Face recognition using unsupervised feature extraction," in Proc. Int. Neural Network Cant, 1990.

[22] I. Craw, H. Ellis, and J.R. Lishman, "Automatic extraction of face features," Patt. Recogn.Lett.,vol. 5, pp. 183-187, Feb. 1987.

[23] W. W. Bledsoe, "Man-machine facial recognition,” Tech. Rep. PRI:22,Panoramic Res. Inc., Palo Alto, CA, 1966.

[24]Yoshida, K., Kamruzzaman, M., Jewel, F.A. and Sajal, R.F., 2007, December. Design and implementation of amachine vision based but low cost stand alone system for real time counterfeit Bangladeshi bank notesdetection. In Computer and information technology, 2007.iccit 2007.10th international conference on (pp. 1-5).IEEE. 